\title{
Interaction of quasilocal harmonic modes and boson peak in glasses
}

\author{
D. A. Parshin ${ }^{1,2}$ and C. Laermans ${ }^{1}$ \\ ${ }^{1}$ Department of Physics, Katholieke Universiteit Leuven, Celestijnenlaan 200D, B-3001 Leuven, Belgium \\ ${ }^{2}$ St.Petersburg State Technical University, 195251, Polytechnicheskaya 29, St.Petersburg, Russia
}

(November 20, 2018)

\begin{abstract}
The direct proportionality relation between the boson peak maximum in glasses, $\omega_{b}$, and the IoffeRegel crossover frequency for phonons, $\omega_{d}$, is established. For several investigated materials $\omega_{b}=$ $(1.5 \pm 0.1) \omega_{d}$. At the frequency $\omega_{d}$ the mean free path of the phonons $l$ becomes equal to their wavelength because of strong resonant scattering on quasilocal harmonic oscillators. Above this frequency phonons cease to exist. We prove that the established correlation between $\omega_{b}$ and $\omega_{d}$ holds in the general case and is a direct consequence of bilinear coupling of quasilocal oscillators with the strain field.
\end{abstract}

61.43.Fs, 63.50+x, 78.30.Ly

The properties of harmonic vibrational excitations in disordered media and glasses become now a very active topic of scientific research [1]. Contrary to the quite well established behavior of electrons in disordered conductors there is no consensus at all regarding the harmonic vibrations in ordinary glasses. The most common and challenging of their signatures is the so-called boson peak observed in numerous experiments in the low-frequency Raman and inelastic neutron scattering. The physical origin of the peak is however still a matter of great debates. The common view is that the solution of this problem is a corner-stone for our veritable understanding of glassy vibrational dynamics.

The main discussion in the literature involves now the question whether the harmonic vibrations responsible for the boson peak are propagating plane waves (phonon like) [2 or localized because of disorder [3]. The third possibility, which we share in this paper, is that they are neither propagating waves nor localized but have a diffusive nature [4].

For the solution of this crucial question quite powerful and expensive experimental techniques are now in use. First of all these are Raman experiments themselves. But since visible light due to energy and momentum conservation laws does not interact properly with sound-like excitations, one has to use two other possibilities, namely inelastic X-ray and neutron scattering.

The main difficulty in the inelastic X-ray scattering experiments is the very high incident photon energy $(\simeq 20$ $\mathrm{KeV}$ ), and its relatively small change, of the order of $10^{-7}$. As a result, the "X-ray boson peak" is superimposed on the steep wings of strong elastic line in the forward direction. Therefore, a serious problem arises to correctly resolve it and answer the crucial question whether it changes with the momentum transfer.

This difficulty explains why recently two independent groups using the same experimental setup but different fitting procedures have arrived to completely opposite conclusions about the propagating character of the exci- tations in vitreous $\mathrm{SiO}_{2}$ at the boson peak range [2, 3]. The situation with inelastic neutron Brillouin scattering (for small momentum transfer) is not much better.

To solve this problem from our point of view it is necessary to separate the Brillouin (phonon) lines of the boson peak (as in the usual light scattering experiments). Then increasing the momentum transfer $\mathbf{q}$ one can observe how the Brillouin line shifts and broadens approaching the boson peak from the low frequency side. This scenario implies the existence in the $q$ range $0.1 \div 1 \mathrm{~nm}^{-1}$ of $t w o$ peaks, one from damped phonons (Brillouin line) and another the boson peak itself. However such experiments will hardly appear in a nearest future.

Therefore at this stage of our knowledges any theoretical insight into the problem would help a lot to establish the true picture of the harmonic vibrations in glasses. The main idea is that the boson peak, being a universal glassy property, should be related with other universal properties of glasses.

In the papers [5] the idea was put forward that the boson peak corresponds to the Ioffe-Regel crossover frequency for phonons, $\omega_{d}$. As it was conjectured in [7] above $\omega_{d}$ phonons cease to exist as well defined plane wave excitations. Later this idea was adopted (supported) in many papers [3,8], though taken alone without any theory, it of course could not explain why actually this correlation occurs.

The explanation of such a correlation based on the theory of soft atomic potentials in glasses [9] was proposed in [7]. The main idea is that at this frequency the dipole-dipole interaction between quasilocal harmonic oscillators renormalizes their density of states (DOS) from the bare value $g(\omega) \propto \omega^{4}$ (for independent oscillators) to the $g(\omega) \propto \omega$ (for coupled oscillators) behavior. As a result a boson peak appears in $g(\omega) / \omega^{2}$ at a frequency $\simeq \omega_{d}$. The reason for the DOS transformation is a strong level repulsion in the course of the interaction of oscillators. Above $\omega_{d}$ the oscillators become delocalized [10].

This correlation was checked indirectly for $A s_{x} S e_{1-x}$ 
glasses [6] and excellent agreement was found between the position of the bump in the reduced specific heat, $C(T) / T^{3}$ (prototype of the boson peak), and the energy $E_{d}=\hbar \omega_{d}$ for different $x$ compositions. The direct evidence should of course include the comparison of the boson peak and the Ioffe-Regel crossover frequency which is explicitly calculated from the mean free path of phonons $l$. In its turn $l$ can be easily evaluated from the fit of the thermal conductivity data which are available for many glasses. The purpose of our paper is just to make such a comparison.

In this paper on the basis of experimental data analysis for different glasses we have established for a first time a direct proportionality relation between the position of the boson peak and the Ioffe-Regel crossover frequency $\omega_{d}$. At this frequency the phonon mean free path with respect to the resonant scattering on quasilocal harmonic oscillators becomes equal to the wave length. To proceed further we should briefly remind the main theoretical and experimental results in this field.

It is well known that for many low-temperature properties of glasses two-level systems (TLS's) and phonons are responsible [11]. However, at higher frequencies it was well established that there is an excess of additional low frequency harmonic modes which dominate the specific heat above a few Kelvin [12,13]. They also strongly reduce the phonon mean free path producing the plateau in the thermal conductivity of glasses [14.

In Ref. [5] it was argued that these excess modes are quasilocal soft harmonic oscillators (HO) coexisting with TLS's (and phonons) with density of states increasing as $g(\omega) \propto \omega^{4}$ at moderate frequencies $\left(W / \hbar<\omega \leq \omega_{d}\right.$, see below). As a result with rising the temperature the linear temperature behavior of the specific heat (TLS's contribution) changes to $C(T) \propto T^{5}$ dependence. Such crossover results in a minimum in $C(T) / T^{3}$, at some $T_{\min } \approx 0.5-3 \mathrm{~K}$ [5, 16 .

The quasilocal vibrations open also a new effective channel for the phonon scattering. Their steeply rising DOS, $g(\omega)$, leads to the same frequency dependence of the inverse mean free path for phonons because of resonant scattering on these oscillators 14

$$
l_{\mathrm{res}, \mathrm{HO}}^{-1}=\frac{\pi}{6 \sqrt{2}} \frac{C \omega}{v}\left(\frac{\hbar \omega}{W}\right)^{3} \propto \omega^{4} .
$$

This dependence looks like elastic Rayleigh scattering from glass inhomogeneities though the physical mechanism is drastically different. We believe that in ordinary glasses the Rayleigh scattering is small in comparison with this contribution.

Except for the sound velocity $v$ there are only two parameters in (11). The first one is a characteristic energy $W$ which is related with the position of the minimum in $C(T) / T^{3}, W \approx 2 k T_{\min }$ [5] [16]. The second is a coupling constant $C$ which describes the relative change of $v$ with
TABLE I. Boson peak frequency, $\omega_{b}$, coupling constant of longitudinal phonons with TLS's, $C_{l}$, and $T_{\min }$ for several glasses.

\begin{tabular}{llll}
\hline \hline Glass & $\begin{array}{l}T_{\min } \\
(\mathrm{K})\end{array}$ & $\begin{array}{l}\omega_{b} \\
\left(\mathrm{~cm}^{-1}\right)\end{array}$ & $\begin{array}{l}C_{l} \\
\left(10^{-4}\right)\end{array}$ \\
\hline $\mathrm{SiO}_{2}$ & ${ }^{a} 2.1$ & ${ }^{b} 52$ & ${ }^{c} 3.1$ \\
$\mathrm{As}_{2} \mathrm{~S}_{3}$ & ${ }^{d} 0.8$ & ${ }^{f} 26$ & ${ }^{c, g} 1.6$ \\
$\mathrm{~B}_{2} \mathrm{O}_{3}$ & ${ }^{a} 1$ & ${ }^{h} 28$ & ${ }^{i} 2.4$ \\
$\mathrm{Se}$ & ${ }^{a} 0.6$ & ${ }^{j} 18$ & ${ }^{c} 1.2$ \\
$\mathrm{PS}$ & ${ }^{k} 0.9$ & ${ }^{l} 17$ & ${ }^{c} 3.6$ \\
$\mathrm{GeO}_{2}$ & ${ }^{m} 1.75 ; 2$ & ${ }^{b} 45$ & ${ }^{n} 2.5$ \\
$\mathrm{LaSF}-7$ & $2.5(?)$ & ${ }^{o} 80$ & ${ }^{c} 1.2$ \\
$\mathrm{LiCl} \cdot 7 \mathrm{H}_{2} \mathrm{O}$ & ${ }^{a} 3.3$ & ${ }^{p} 60$ & ${ }^{c} 7.2$ \\
\hline \hline
\end{tabular}

${ }^{a}$ Ref. 117, ${ }^{b}$ Ref. 18], ${ }^{c}$ Ref. [19, ${ }^{d}$ Ref. 20, ${ }^{f}$ Ref. [21], ${ }^{g}$ Ref. [22], ${ }^{h}$ Ref. [23], ${ }^{i}$ Ref. |24], ${ }^{j}$ Ref. [25], ${ }^{k}$ Ref. $\left.{ }^{26}\right]$, ${ }^{l}$ Ref. [27], ${ }^{m}$ Ref. [28], ${ }^{n}$ Ref. [29], ${ }^{o}$ Ref. [30], ${ }^{p}$ Ref. [31].

temperature due to resonance scattering of phonons on TLS's [11]

$$
(\Delta v / v)_{\mathrm{res}, \mathrm{TLS}}=C \ln \left(T / T_{0}\right) .
$$

Both experimental values of $C$ and $T_{\min }$ are well known for many glasses. It gave the unique possibility, without any fitting parameters using (11), to successfully reproduce the correct value of the thermal conductivity plateau for vitreous $\mathrm{SiO}_{2}$ and $\mathrm{Se}$ 14 and for some other glasses as well [15]. From this point of view one can consider Eq.(11) to be in a good agreement with the thermal conductivity data.

Using (11) one can easily obtain the expression for the Ioffe-Regel crossover frequency $\omega_{d}$ [6, 6 ]

$$
\hbar \omega_{d}=0.75 W C^{-1 / 3}
$$

For example for $v-\mathrm{SiO}_{2}, \mathrm{C}_{l}=3.1 \times 10^{-4}, \mathrm{~W} / k=4.2 \mathrm{~K}$ and $\hbar \omega_{d} / k \approx 47 \mathrm{~K}$.

Now we can proceed to the main aim of our paper and check whether there exists a correlation between the boson peak frequency $\omega_{b}$ and $\omega_{d}$ determined by (3). For that purpose we collected in the Table If all the necessary experimental parameters $T_{\min }, C_{l}$ and $\omega_{b}$ for several glasses. The two different values of $T_{\min }$ for $a-\mathrm{GeO}_{2}$ correspond to two different samples investigated in [28]. The value of $T_{\min }$ for LaSF-7 glass is a reasonable guess value (we did not find it in a literature).

Now using the relation $W=2 k T_{\min }$ and Eq. 3 we can calculate $\omega_{d}$ for longitudinal phonons and compare it with $\omega_{b}$. The result of this comparison is shown on Fig.1. One can see from the figure that all the data lies near a straight line which has the slope equal to 1.53 . It means that within the experimental accuracy there is a direct proportionality relation between the boson peak maximum $\omega_{b}$ and the Ioffe-Regel crossover frequency for phonons $\omega_{d}$. 
In what follows we are going to prove that this correlation results in fact from the bilinear coupling between quasilocal oscillators and the deformation field $\varepsilon$

$$
\mathcal{H}_{\text {int }}=\Lambda x \varepsilon
$$

proposed in 14. In this sense it can be regarded as a rather general rule.

With the use of (任) one can easily show that the elastic interaction between two oscillators has a dipole-dipole character

$$
V_{\mathrm{int}} \simeq \frac{\Lambda^{2}}{\rho v^{2} r_{i j}^{3}} x_{i} x_{j}
$$

with $\rho$ being the mass density of the glass.

Suppose that the DOS of noninteracting oscillators is $g(\omega)$. Then making use of (4) and the Golden rule one can calculate in the usual way the phonon mean free path due to the resonance scattering on these oscillators

$$
l_{\text {res, } \mathrm{HO}}^{-1}=\frac{\pi \Lambda^{2}}{2 M \rho v^{3}} g(\omega)
$$

where $M$ is an oscillator mass. It is important that this scattering has the same frequency dependence as the bare density $g(\omega)$. The Ioffe-Regel crossover frequency $\omega_{d}$ can be estimated from the equation

$$
\left.\frac{g(\omega)}{\omega}\right|_{\omega_{d}} \simeq \frac{M \rho v^{2}}{\Lambda^{2}} .
$$

In order to determine a position of the boson peak we should consider the interaction between resonant oscillators. The concentration of such oscillators with frequencies $\omega$ lying in a small interval $\delta \omega$ around $\omega$ is given by the product $g(\omega) \delta \omega$. Therefore the non-diagonal transition matrix element of the coupling between the two oscillators (5) is equal

$$
\Delta J \equiv\left\langle n_{i+1}, n_{j}\left|V_{\mathrm{int}}\right| n_{i}, n_{j+1}\right\rangle \simeq \frac{\hbar \Lambda^{2} g(\omega)}{2 M \rho v^{2} \omega} \delta \omega .
$$

The physical meaning of this quantity is that it gives a characteristic value for a level repulsion of two resonant oscillators because of their interaction.

If $\Delta J<\hbar \delta \omega$ the level repulsion is weak and the interaction does not change $g(\omega)$. If $\Delta J>\hbar \delta \omega$ the level repulsion is strong and renormalizes the original density [7. The new DOS can be found dividing the oscillator concentration in the interval by the characteristic value of their level repulsion

$$
\widetilde{g}(\omega) \simeq \frac{g(\omega) \delta \omega}{\Delta J / \hbar} \simeq \frac{M \rho v^{2}}{\Lambda^{2}} \omega .
$$

We see that the result is a linear function of $\omega$ which depends only on material parameters of the glass but does not depend on the bare density $g(\omega)$.
In the case when the reduced bare DOS $g(\omega) / \omega^{2}$ increases with $\omega$ we will have a boson peak at some crossover frequency from weak to strong coupling. This frequency is determined by the equation $\Delta J=\hbar \delta \omega$. One can easily see that this equation coincides with equation (7) for $\omega_{d}$. This explains why $\omega_{d}$ correlates with the position of the boson peak in glasses.

The resulting linear $\omega$ dependence of vibrational DOS above the boson peak is a very important and general feature of a system of interacting harmonic oscillators with bilinear dipole-dipole coupling (5). It is a direct consequence of linear $\omega$ dependence of $\left|x_{n, n \pm 1}\right|^{-2}=2 M \omega / \hbar$ and therefore can be regarded as universal. It holds for any bare $g(\omega)$ of quasilocal harmonic modes which rises with frequency faster then $\omega^{2}$. It was observed in experiments on inelastic neutron scattering for amorphous polymers [32], $a-\mathrm{SiO}_{2}$ [33] and $a-\mathrm{GeSe}_{2}$ [34].

The picture described above implies delocalization of the harmonic excitations above the boson peak though they are not supposed to be plane waves. This is also consistent with existing numerical results. The fact that the Ioffe-Regel criterion does not mean localization for vibrational excitations in disordered system was acknowledged in the computer analysis of the percolation network [35]. It was found that vibrational modes above the IoffeRegel crossover give substantial contribution to the heat transfer and have some diffusive nature.

Similar results were obtained in [36] for $a-\mathrm{SiO}_{2}$ where all modes with $\hbar \omega$ between $5-110 \mathrm{meV}$ were found to be delocalized (just to the right of the boson peak). For amorphous silicon it was found [37 that only $3 \%$ of vibrational modes (above $70 \mathrm{meV}$ ) are localized and do not participate in the heat transfer. The remaining $97 \%$ of the modes are delocalized and the majority of them $(93 \%)$ are not plane waves.

In numerical experiments [38] the truly localized modes were discovered at the very end of the frequency spectrum. It is worthwhile to mention that at low frequencies the calculated DOS has a part with linear $\omega$ dependence just in the region of delocalized states. The same results were obtained for amorphous selenium [39]. The modes with frequencies above $0.5 \mathrm{THz}$ were delocalized (again just above the boson peak for this glass).

To conclude, we have established for the first time the correlation between the boson peak frequency and the Ioffe-Regel crossover frequency. We proved that this correlation occurs because of the bilinear coupling between sound waves and quasilocal harmonic vibrations in glasses.

One of the authors (D.A.P.) gratefully acknowledge pleasant hospitality and financial support of the Catholic University of Leuven where this work was done. One of the authors (C.L.) thanks the Belgian FWO for financial support. 
[1] see for example special issue of Phil.Mag., 79, N 11/12 (1999), the Proceedings of 7th International Workshop on Disordered Systems, Molveno, Italy, ed. A.Fontana and G. Viliani.

[2] P. Benassi et al., Phys.Rev.Lett., 77, 3835-3838 (1996).

[3] M. Foret et al., Phys.Rev.Lett., 77, 3831-3834 (1996).

[4] P.B. Allen, and J.L. Feldman, Phys.Rev.B, 48, 12581 (1993).

[5] M.A. Il'in, V.G. Karpov, and D.A. Parshin, Zh.Eksp. Teor.Fiz., 92, 291 (1987) [Sov.Phys.JETP, 65, 165 (1987)].

[6] D.A. Parshin, X. Liu, O. Brand, and H.v. Löhneysen, Z.Phys.B, 93, 57 (1993).

[7] V.L. Gurevich, D.A. Parshin, J. Pelous, and H.R. Schober, Phys.Rev.B, 48, 16318 (1993).

[8] M. Foret et al., Phys.Rev.Lett., 81, 2100 (1998); E. Rat et al., Phys.Rev.Lett., 83, 1355 (1999);

[9] V.G. Karpov, M.I. Klinger, and F.N. Ignat'iev, Zh.Eksp. Teor. Fiz., 84, 760 (1983) [Sov.Phys.JETP, 57, 439 (1983)]; for review see D.A. Parshin, Phys.Sol.State, 36, 991 (1994).

[10] This explains subscript letter " $d$ " in $\omega_{d}$. By term of "delocalization" we understand that being excited harmonic oscillator most probably transfer its energy not to a phonon (in such a case it would be a quasilocal oscillator) but to another oscillator.

[11] W.A. Phillips, Rep.Prog.Phys., 50, 1657 (1987).

[12] U. Buchenau, N. Nücker, and A.J. Dianoux, Phys.Rev. Lett., 53, 2316 (1984).

[13] U. Buchenau et al., Phys.Rev.B, 34, 5665 (1986).

[14] U. Buchenau, Yu.M. Galperin, V.L. Gurevich, D.A. Parshin, M.A. Ramos, and H.R. Schober, Phys.Rev.B, 46, 2798 (1992).

[15] M.A. Ramos, and U. Buchenau, Phys.Rev.B, 55, 5749 (1997).

[16] U. Buchenau et al., Phys.Rev.B, 43, 5039 (1991).

[17] L. Gil et al., Phys.Rev. Lett., 70, 182, 1993.

[18] R.H. Stolen, Phys.Chem.Glasses, 11, 83, 1970.

[19] J.F. Berret, and M. Meißner Z.Phys.B, 70, 65, 1988.

[20] R. Stephens, Phys.Rev.B, 13, 852, 1976.

[21] R.J. Nemanich, Phys.Rev.B, 16, 1665, 1977.

[22] C. Laermans et al., The Physics of Non-Crystalline Solids, Ed. G.H. Frischat, 4 International Conference, p.562, 1977.

[23] V.K. Malinovsky et al., Europhys.Lett., 11, 43, 1990.

[24] D.A. Parshin, Phys.Rev.B, 49, 9400, 1994; M. Devaud, and J.-Y. Prieur, J.Phys.(Paris) Colloq., 43, C9-497, 1982; S.Rau, C. Enns, and S. Hunklinger, Phys.Rev.B, 52, 7179 (1995).

[25] V.N. Novikov, and A.P. Sokolov, Sol.State Comm., 77, 243, 1991.

[26] R.C. Zeller, and R.O. Pohl, Phys.Rev.B, 4, 2029, 1971.

[27] A.P. Sokolov, private communication.

[28] A.P. Jeapes et al., Phil.Mag., 29, 803, 1974.

[29] C. Laermans, V. Keppens, and R.Weeks, Phys.Rev.B, 55, 2701, 1997.

[30] J.L. Prat, F. Terki, and J. Pelous, Phys.Rev.Lett., 77, 755, 1996.

[31] K.G. Breitschwerdt, and S. Gut, Proc. 12 Intern. Conf. on acoustic, Toronto,1986, p. G2-6.

[32] U. Buchenau, Phil.Mag.B, 71, 793 (1995).

[33] A. Wischnewski et al., Phil.Mag.B, 77, 579 (1998).

[34] R.L. Cappelletti et al., Phys.Rev.B, 52, 9133 (1995).

[35] P. Sheng, M. Zhou, and Z.-Q. Zhang, Phys. Rev. Lett., 72, 234 (1994).

[36] W. Jin et al., Phys. Rev. B, 48, 9359 (1993).

[37] J.L. Feldman et al., Phys. Rev. B, 48, 12589 (1993); P.B. Allen et al., p.1715 in Ref 11.

[38] H.R. Schober, and B.B. Laird, Phys.Rev.B, 44, 6746 (1991); B.B. Laird, and H.R. Schober, Phys. Rev. Lett 66, 636 (1991).

[39] C. Oligschleger, and H.R. Schober, Physica A, 201, 391 (1993).

Figure caption

Fig.1 Position of the boson peak $\omega_{b}$ versus the IoffeRegel crossover frequency $\omega_{d}$ for several glasses. 


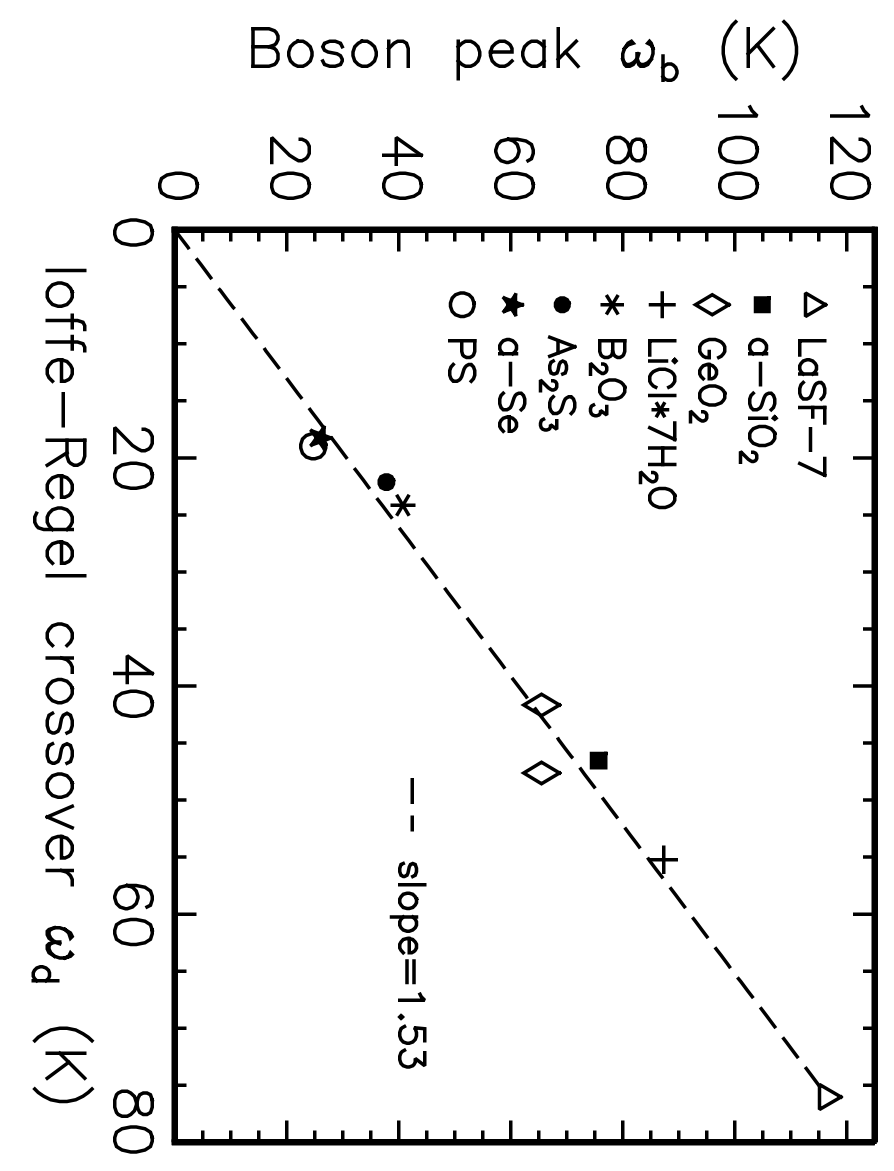

